\title{
Are There Different Viewpoints About the Management of Type 2 Diabetes Mellitus and Comorbidities? A Multidisciplinary Spanish Qualitative Research
}

\author{
Francesc-Xavier Cos (D) · Ricardo Gómez-Huelgas (D) · Fernando Gomez-Peralta (D)
}

Received: October 14, 2021 / Accepted: November 26, 2021 / Published online: December 20, 2021

(c) The Author(s) 2021

\begin{abstract}
Introduction: The aim of this study was to explore the vision of a large multidisciplinary group of physicians treating type 2 diabetes mellitus (T2DM) in Spain, with a special focus on controversial management aspects. The perceptions of primary care (PC) physicians and hospital care (HC) specialists were compared.

Methods: This was a mixed survey that included Delphi-like statements and opinion, attitude and behaviour (OAB) questions. The Delphi-like statements were assessed on the basis of the degree of agreement among respondents, and a
\end{abstract}

F.-X. Cos

Medicina Familiar y Comunitaria, Fundación Instituto Universitario para la Investigación en Atención Primaria de Salud Jordi Gol i Gurina (IDIAPJGol), Innovation Officer, Institut Català de la Salut., Centro de Atención Primaria Sant Martí de Provençals, Barcelona, Spain e-mail: francescxaviercos@gmail.com

R. Gómez-Huelgas $(\bowtie)$

Servicio de Medicina Interna, Hospital Regional Universitario, Instituto de Investigación Biomédica de Málaga (IBIMA), Universidad de Málaga (UMA), Málaga, CIBER Fisiopatología de Obesidad y

Nutrición (CIBERobn), Instituto de Salud Carlos III, Madrid, Spain

e-mail: ricardogomezhuelgas@hotmail.com

F. Gomez-Peralta

Unidad de Endocrinología y Nutrición, Hospital

General de Segovia, Segovia, Spain

e-mail: fgomezperalta@gmail.com descriptive analysis was performed on the answers to the $\mathrm{OAB}$ questions.

Results: A total of 296 participants responded to the first wave of the survey, of whom 293 responded to the second wave ( 211 from PC and 80 from HC, with two respondents for whom there were no data on specialty). A high degree of consensus $(\mathrm{CNS} \geq 0.8)$ was obtained in all the statements. A proactive approach to detect prediabetes or T2DM in asymptomatic people was highly supported $(80.4 \%$ of agreement). Introducing early treatment intensification was considered to favour the durability of glycaemic control and to delay the progression of the disease $(80.4 \%)$. There was agreement on the statement that glycaemic variability constitutes a risk factor for chronic complications, although differences in the perceptions of HC physicians and PC specialists were identified (86.3 vs. $80.1 \%$, respectively). More HC physicans than PC specialists considered comorbidities to affect the ability to self-care (95 vs. $82.9 \%$, respectively).

Conclusions: The survey revealed that there was a high, albeit not universal, degree of agreement amongst PC physicians and HC specialists in relation to prevention, screening and diagnosis of T2DM; early treatment intensification; dysglycaemias; and the management of patients with comorbidities. The statement on the management of patients with comorbidities elicited the highest difference between PC physicans and HC specialists. The results of this survey indicate that there is room for 
improvement in terms of implementing strategies in these areas.

Keywords: Antidiabetic agents; Clinical guidelines; Comorbidities; Diabetes Mellitus, type 2; Drug therapy, combination; Prediabetes; Primary care; Qualitative research

\section{Key Summary Points}

Why carry out this study?

Type 2 diabetes mellitus (T2DM) is a complex disease with a large number of complications, and its management involves several specialists in addition to primary care (PC) physicians, and thus different approaches.

Knowledge and a correct approach regarding screening, prevention, diagnosis, early treatment intensification, dysglycaemia and comorbidities are crucial to avoid future complications in patients with T2DM.

The aim of the study was to analyse the perception of a large group of physicians involved in T2DM management on these subjects, and to identify differences between the perceptions of PC physicians and hospital care specialists.

\section{What has been learned from the study?}

This study shows that there is a high level of agreement amongst participants but that there is still room for improvement in terms of implementing strict glycaemic control, individualizing glycaemic control goals, and indication of early treatment intensification, mostly amongst PC physicians.

Further studies aimed at illuminating potential behavioural differences between different medical specialties in clinical practice would help to detect existing mismatches between knowledge and clinical behaviour regarding the management of T2DM.

\section{INTRODUCTION}

Although the pathophysiological changes that occur in patients with type 2 diabetes mellitus (T2DM) commonly remain undetected for several years before the disease is detected, i.e. during the long and asymptomatic pre-clinical phase of the disease, complications may have developed by the time of diagnosis [1]. Consequently, early detection of T2DM and the implementation of early and intensive interventions are relevant not only to prevent betacell dysfunction but also to intervene before the blood glucose thresholds currently set for T2DM diagnosis are reached to protect against potential cardiovascular (CV) risk factors [2, 3]. CV disease (CVD) accounts for one half of all T2DM-related deaths [4], and to address this issue, glucoselowering drugs that have been developed over the past decade have been tested in CV outcomes trials that have included patients at high risk of $\mathrm{CV}$ disease, with the aim to evaluate CV endpoints [5]. Likewise, the most recent international recommendations have intensively focused on CVD to guide T2DM management, whilst other comorbidities and patient preferences been minimized despite that almost 80\% of patients with T2DM have comorbidities other than CVD [6]. T2DM increases the risk of not only coronary artery disease, peripheral arterial disease and stroke, but also kidney failure, nontraumatic amputations and blindness [7-9].

Early treatment intensification strategies have been demonstrated to provide significant reductions in glycated haemoglobin (HbA1c) over both the short [6] and long term [10]. Moreover, a recent study comparing an early combination of vildagliptin plus metformin with metformin alone also showed a reduction in the time to treatment failure in the long term (5-year treatment period) in patients with recent T2DM and HbA1c levels of 6.5-7.5\% [10]. This recent evidence has prompted an update of the recommendations in this regard [11, 12], indicating alternative strategies to keeping to the traditional sequential therapeutic approach of initiating treatment with metformin and adding a second hypoglycaemic agent only when glycaemic objectives are not achieved 
[13], a clinical strategy often related to therapeutic inertia and lack of glycaemic control [14-17].

The complexity of T2DM and the variability and severity of its complications translate into the involvement of several specialists in the management of the disease and, thereby, into the convergence of different approaches. A wide variety of clinical guidelines exist in the field, although conformance and adherence to them are not always optimal, and clinical outcomes and related costs might be compromised [18-21].

The objective of the current study was to analyse the perception of a large group of physicians who are mainly involved in this area on certain controversial aspects of the management of T2DM, such as prevention, screening, diagnosis, early treatment intensification and management of dysglycaemia and chronic complications, as well as to identify differences between primary care (PC) physicians and hospital care (HC) specialists.

\section{METHODS}

A dedicated scientific committee consisting of three opinion leaders designed a survey to cover a number of issues covered in clinical practice guidelines. These individuals had different specialties (internal medicine, endocrinology and PC) and had wide clinical and academic expertise, as well as a broad spectrum of publications on T2DM. The survey included 25 Delphi-like statements and 13 questions on opinion, attitude and behaviour (OAB). Here, we report the results on a selection of 14 statements and five $\mathrm{OAB}$ questions. The $\mathrm{OAB}$ questions were either multiple choice or to be answered on a rating scale, and they were formulated within the context of some of the Delphi-like statements.

The survey was provided to a representative sample of 300 physicians from different regions of Spain. The selected participants were PC physicians and HC specialists whose usual practice included the management of T2DM. They were selected by a non-probabilistic directed sampling of convenience by conglomerates, according to proportional geographic and population distribution criteria.
The degree of agreement with the Delphilike statements was evaluated in the first wave of the survey using a 5-point Likert scale (from 'totally disagree' to 'totally agree') and analysed using two metrics: the consensus value (CNS), applying the Tastle technique [22], and the collapse of the five Likert categories into three ('disagree', 'undecided' and 'agree'). The consensus threshold in the Delphi-like statements was CNS $\geq 0.70$. Those statements that did not obtain a high degree of consensus $(\mathrm{CNS}<0.80)$ were re-evaluated in a second wave of the survey, either by modifying or inverting the composition of the statements. The percentages of answer were calculated for the multiple choice $\mathrm{OAB}$ questions, and the mean value was calculated for the rating scale $\mathrm{OAB}$ questions. The Mann-Whitney $U$ test was used to evaluate differences obtained between the answers of PC physicians and HC specialists.

\section{Compliance with Ethics Guidelines}

The study was based on an on-line survey that did not require data on individual patients to be recorded nor involve the participation of patients. There was no evaluation of any specific medication as the main factor. Therefore, this study did not require ethical approval as none of the criteria of post-authorization studies (PASS, non-interventional PASS) covered by the Spanish Agency for Medicines and Health Products (AEMPS) were met. Data were collected by means of anonymous questionnaires in online format, completed by physicians in accordance with their usual practice. Participation was voluntary. The respondents expressed their consent to participate in the survey through logging into the secure online survey platform and actively clicking a consent box.

\section{RESULTS}

\section{Participants}

A total of 296 participants responded to the first wave of the survey, of whom 293 responded to the second wave (211 from PC physicians and 
80 from HC specialists; for two responders there were no data on specialty). In terms of age, $21.6 \%$ of the participants were $\leq 45$ years old, $31.0 \%$ were aged $46-55$ years and $47.7 \%$ were older than 56 years. Of the participants, $65.5 \%$ were men and $67.9 \%$ had been in medical practice for $\geq 20$ years. Regarding treatment protocols, $74.4 \%$ of the participants declared following the recommendations of clinical practice guidelines for the diagnosis and followup of T2DM, and $21.0 \%$ declared that they followed PC or hospital protocols.

\section{Delphi Statements}

After the second wave, the CNS reached a value of $\geq 0.80$ for all of the statements included in the survey (Table 1). When responses were collapsed to three categories, similar results were obtained (Fig. 1).

Opportunistic screening strategies for the early diagnosis in asymptomatic patients were considered to be beneficial by $82.8 \%$ of participants. Similarly, $80.4 \%$ of participants considered periodic laboratory tests to be a convenient strategy for screening of prediabetes/diabetes in asymptomatic subjects. Agreement amongst participants was especially high regarding the consideration of lifestyle interventions as factors that delay or prevent the progression of prediabetes to diabetes $(90.7 \%)$, and in the periodic determination of HbA1c to prevent long-term complications of T2DM (88.7\%) (Fig. 1a).

Early strict glycaemic control was considered by $88.0 \%$ of participants as a means to reduce the prevalence of chronic complications and/or prevent disease progression. Slightly more than $95.0 \%$ of participants agreed that detection and control of hyperglycaemia in the asymptomatic stages of T2DM affects the prevention of complications, and $80.4 \%$ agreed that early intensification with combined therapy provides greater and longer-lasting benefits in terms of glycaemic control and delay of disease progression (Fig. 1b).

Glycaemic variability was agreed upon as a risk factor for chronic complications in and of itself (91.8\% of participants), and postprandial hyperglycaemia was considered by $81.8 \%$ of participants to be a CV risk factor. The potential risk of severe hypoglycaemia was considered a key parameter for establishing the objectives of control and pharmacological management therapies of T2DM by $90.7 \%$ of participants (Fig. 1c).

The presence of chronic complications and comorbidities was considered to hinder selection between the different therapeutic options and combinations by $84.0 \%$ of participants. That comorbidities should be considered to affect the ability to self-care was agreed upon by $86.3 \%$ of participants, and $86.9 \%$ of participants were in agreement that psychiatric disorders affected antidiabetic treatment. The necessity of screening for corticosteroid-induced hyperglycaemia in patients with T2DM-COPD who receive medium or high doses of corticosteroids to treat the exacerbations was also agreed to by a high proportion of participants (91.1\%) (Fig. 1d).

\section{OAB Questions}

A long life expectancy, good functional and cognitive status and a recent diagnosis of T2DM were chosen as factors that prompt individualization for stricter glycaemic control goals by more than $70.0 \%$ of the participants (Fig. 2a).

In patients lacking metabolic control despite the prescription of adequate treatment, the reasons for therapeutic failure seem to be multifactorial: comorbidities, functional factors, therapeutic regimen-derived factors and disease-derived factors were considered to be the most contributory factors (Fig. 2b).

Nearly $70.0 \%$ of participants expressed the view that independently of age, basal glycaemic control values are the main driver for treatment intensification (Fig. 3).

Among all participants, $85.8 \%$ declared using capillary glycaemia and a symptom diary to detect, monitor and assess the risk of hypoglycaemia, $64.5 \%$ admitted doing so by collecting data and information on the conditions associated with the risk of hypoglycaemia, $29.1 \%$ declared using continuous blood glucose 
Table 1 Consensus degree obtained for Delphi statements

\begin{tabular}{|c|c|c|c|c|}
\hline Statements & $\begin{array}{l}\text { CNS } \\
(n=291)\end{array}$ & $\begin{array}{l}\text { CNS PC } \\
(n=211)\end{array}$ & $\begin{array}{l}\text { CNS HC } \\
(n=80)\end{array}$ & $\begin{array}{l}P \text { value } \\
(\mathrm{MW})\end{array}$ \\
\hline \multicolumn{5}{|l|}{ Prevention, screening and diagnosis } \\
\hline $\begin{array}{l}\text { 1. There are benefits derived from applying an opportunistic screening } \\
\text { strategy for the early diagnosis of T2DM in asymptomatic subjects }\end{array}$ & 0.82 & 0.82 & 0.82 & 0.619 \\
\hline $\begin{array}{l}\text { 2. Periodic laboratory testing (fasting basal glycaemia, HbAlc) is } \\
\text { convenient for the screening of prediabetes/diabetes in asymptomatic } \\
\text { subjects }\end{array}$ & 0.80 & 0.80 & 0.81 & 0.748 \\
\hline $\begin{array}{l}\text { 3. Progression of prediabetes to diabetes can be delayed or prevented } \\
\text { through lifestyle interventions }\end{array}$ & 0.89 & 0.88 & 0.91 & 0.541 \\
\hline $\begin{array}{l}\text { 4. Periodic determination of } \mathrm{HbAlc} \text { contributes to the prevention of } \\
\text { long-term complications in patients with diagnosed T2DM }\end{array}$ & 0.87 & 0.87 & 0.88 & 0.709 \\
\hline \multicolumn{5}{|l|}{ Early treatment intensification } \\
\hline $\begin{array}{l}\text { 5. If performed early, during the first years after diagnosis of T2DM, } \\
\text { strict glycaemic control reduces the prevalence of chronic } \\
\text { complications and/or prevents progression }\end{array}$ & 0.86 & 0.85 & 0.88 & 0.980 \\
\hline $\begin{array}{l}\text { 6. Detection and control of hyperglycaemia in asymptomatic stages of } \\
\text { T2DM affects the prevention of complications }\end{array}$ & 0.93 & 0.92 & 0.95 & 0.661 \\
\hline $\begin{array}{l}\text { 7. A first therapeutic step of early intensification with combined } \\
\text { therapy following a diagnosis of T2DM provides greater and longer- } \\
\text { lasting benefits for patients, by favouring the durability of glycaemic } \\
\text { control and delaying the progression of the disease }\end{array}$ & 0.81 & 0.81 & 0.82 & 0.675 \\
\hline \multicolumn{5}{|l|}{ Dysglycaemia } \\
\hline $\begin{array}{l}\text { 8. Glycaemic variability (oscillation, frequency and intensity of } \\
\text { fluctuations in blood-glucose concentrations) constitutes, in and of } \\
\text { itself, a risk factor for chronic complications in patients with T2DM }\end{array}$ & 0.81 & 0.79 & 0.85 & 0.019 \\
\hline $\begin{array}{l}\text { 9. Postprandial hyperglycaemia constitutes, in and of itself, a CV risk } \\
\text { factor in patients with T2DM }\end{array}$ & 0.80 & 0.79 & 0.82 & 0.421 \\
\hline $\begin{array}{l}\text { 10. The potential risk of severe hypoglycaemia is a key parameter to set } \\
\text { the objectives of control and the pharmacological management of } \\
\text { T2DM }\end{array}$ & 0.89 & 0.88 & 0.93 & 0.097 \\
\hline \multicolumn{5}{|l|}{ Chronic complications and comorbidities } \\
\hline $\begin{array}{l}\text { 11. The presence of chronic complications and comorbidities in the } \\
\text { diabetic patient may hinder the election between the different } \\
\text { therapeutic options and combinations }\end{array}$ & 0.83 & 0.82 & 0.85 & 0.515 \\
\hline 12. Comorbidity affects the patient's ability to self-care & 0.84 & 0.81 & 0.91 & 0.000 \\
\hline $\begin{array}{l}\text { 13. The coexistence of serious psychiatric disorders in patients with } \\
\text { T2DM affects antidiabetic treatment }\end{array}$ & 0.85 & 0.84 & 0.87 & 0.168 \\
\hline
\end{tabular}


Table 1 continued

\begin{tabular}{|c|c|c|c|c|}
\hline Statements & $\begin{array}{l}\text { CNS } \\
(n=291)\end{array}$ & $\begin{array}{l}\text { CNS PC } \\
(n=211)\end{array}$ & $\begin{array}{l}\text { CNS HC } \\
(n=80)\end{array}$ & $\begin{array}{l}P \text { value } \\
\text { (MW) }\end{array}$ \\
\hline $\begin{array}{l}\text { 14. A specific screening for corticosteroid-induced hyperglycaemia } \\
\text { (guidelines for self-measurement of capillary glycaemia) and the } \\
\text { assessment of adjustments for the treatment of hyperglycaemia should } \\
\text { be performed in patients with T2DM-COPD comorbidity, who } \\
\text { receive medium or high doses of corticosteroids to treat the } \\
\text { exacerbations of their pulmonary disease }\end{array}$ & 0.87 & 0.85 & 0.91 & 0.021 \\
\hline
\end{tabular}

monitoring and $12.8 \%$ declared using tests or questionnaires (Fig. 4a).

Excessive insulin dose, a reduction or delay in carbohydrate intake, inappropriate use of oral hypoglycaemic agents and an increase in physical exercise were the situations more commonly considered as potential causes of hypoglycaemia (Fig. 4b).

\section{PC and HC Analysis}

No differences were observed between PC physicans and HC specialists in any statement regarding prevention, screening and diagnosis and early treatment intensification. However, the degree of consensus was significantly higher amongst HC participants (86.3\%) than among PC participants $(80.1 \%)$ in terms of considering glycaemic variability as a risk factor for chronic complications.

The percentage of agreement was significantly higher among HC participants than among PC participants when the statement comorbidities affect the ability to self-care was considered (95.0 vs. 82.9\%, respectively). Differences were also observed on the necessity of screening for corticosteroid-induced hyperglycaemia in the assessment of adjustments for the treatment of hyperglycaemia in patients with T2DM and chronic obstructive pulmonary disease (COPD) who receive medium or high doses of corticosteroids to treat exacerbations, with $95.0 \%$ of HC physicians versus $89.6 \%$ of PC specialists agreeing that such screeing was necessary.

\section{DISCUSSION}

\section{Prevention, Screening and Diagnosis}

Prediabetes is a clinical stage characterized by levels of fasting plasma glucose that are higher than normal but lower than those considered to indicate diabetes. Approximately $5-10 \%$ of patients with prediabetes ultimately develop T2DM each year [23]. Screening strategies, which are often carried out in the PC setting, were agreed upon by the participants in this study as being beneficial and convenient for detecting asymptomatic subjects. A periodic determination of HbA1c was also considered a convenient screening test in asymptomatic subjects and for the prevention of long-term complications in patients who already had a diagnosis of T2DM, as was having a degree of knowledge of the recommendations of the World Health Organization (WHO) [24, 25] and clinical practice guidelines $[13,26]$. In light of the current delayed diagnosis of T2DM in clinical practice, the results reported here highlight the relevance of implementing screening strategies in high-risk individuals, as well as lifestyle interventions, in order to prevent or delay T2DM onset and avoid future 


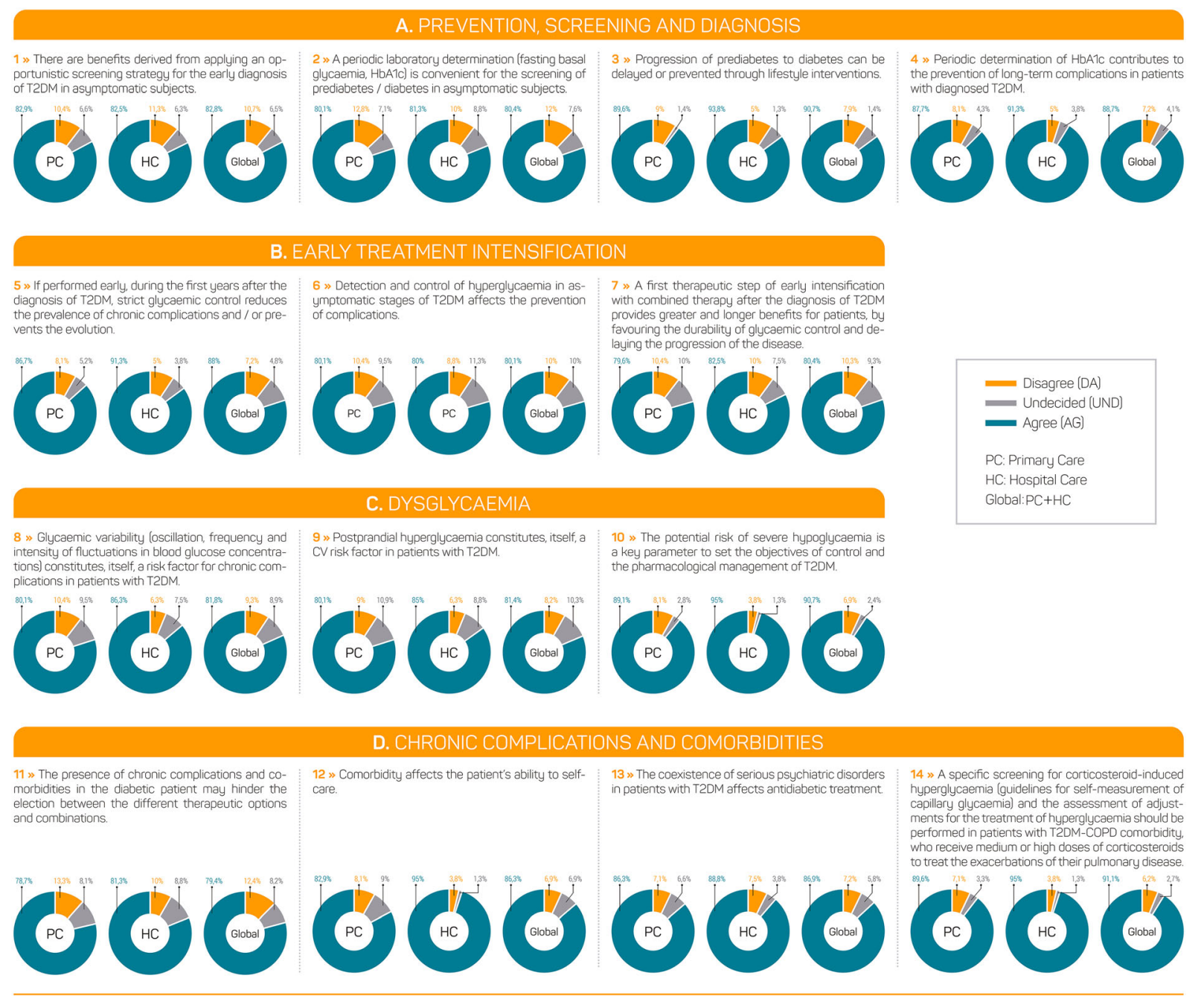

Fig. 1 Percentage of agreement obtained for Delphi statements ( $N=291$ participants)

complications, both of which are more likely to appear in the PC setting.

\section{Early Treatment Intensification and Pharmacological Combination Treatment}

Intensive glycaemic control is inversely related to the risk of microvascular events [27]. Delays in the intensification of treatment for T2DM due to clinical inertia have been shown to increase the risk for CV events [28]. Recent studies have demonstrated the benefits of achieving glycaemic control as early as within the first 12 months from diagnosis [29].
Nonetheless, a cross-sectional epidemiological study in Spain that included patients from 2007 to 2013 found a huge proportion of patients with records of deficient glycaemic control during the last year registered: $55.6 \%$ of patients were receiving monotherapy or no treatment at all, even though $44.8 \%$ presented HbA1c levels $>7.0 \%$ [30]. In our study, agreement on the statement that early treatment intensification following diagnosis is a strategy for providing greater and longer-lasting benefits for patients was high and, remarkably, similar across different PC and HC participants.

Recent international guidelines have included a general recommendation to consider early combination therapy at treatment initiation 
a. In your clinical practice, which factors do influence you to individualize to stricter glycaemic control goals? (multiple choice)

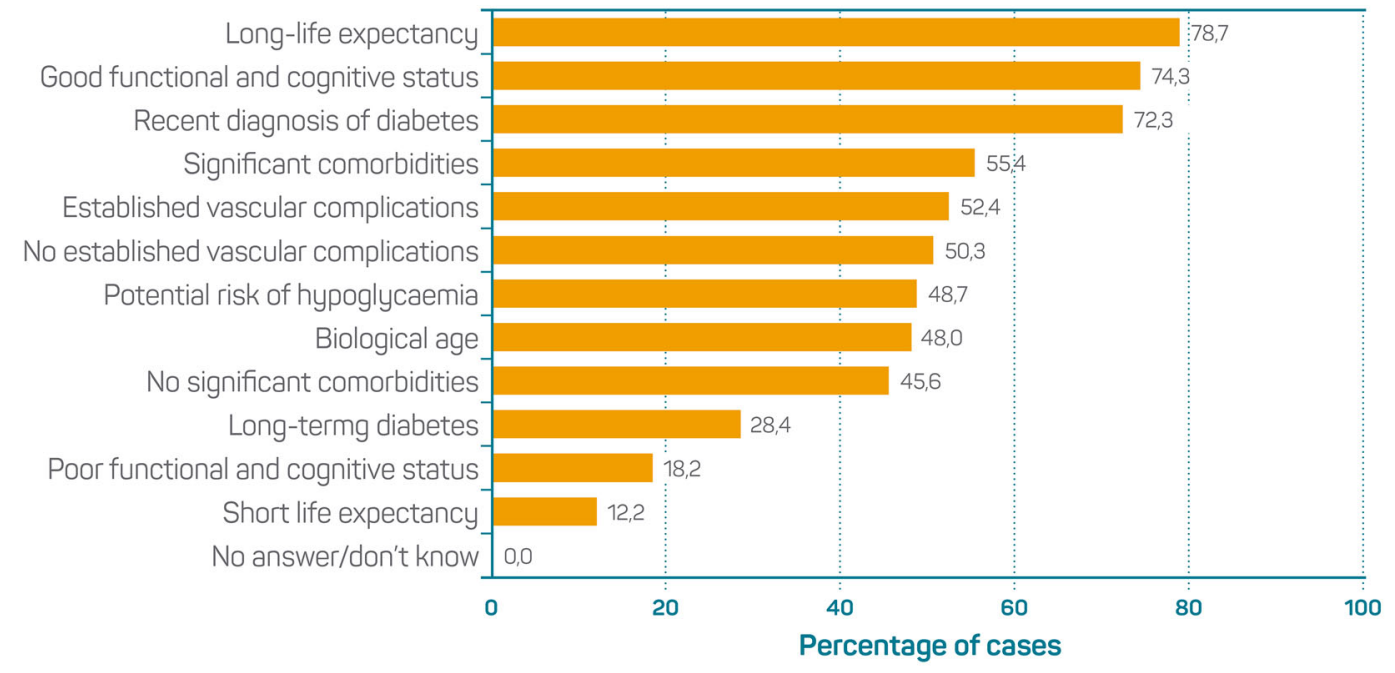

\section{b. In patients with no metabolic control despite adequate treatment, which factors do you consider that contribute to therapeutic failure? (rating scale)}

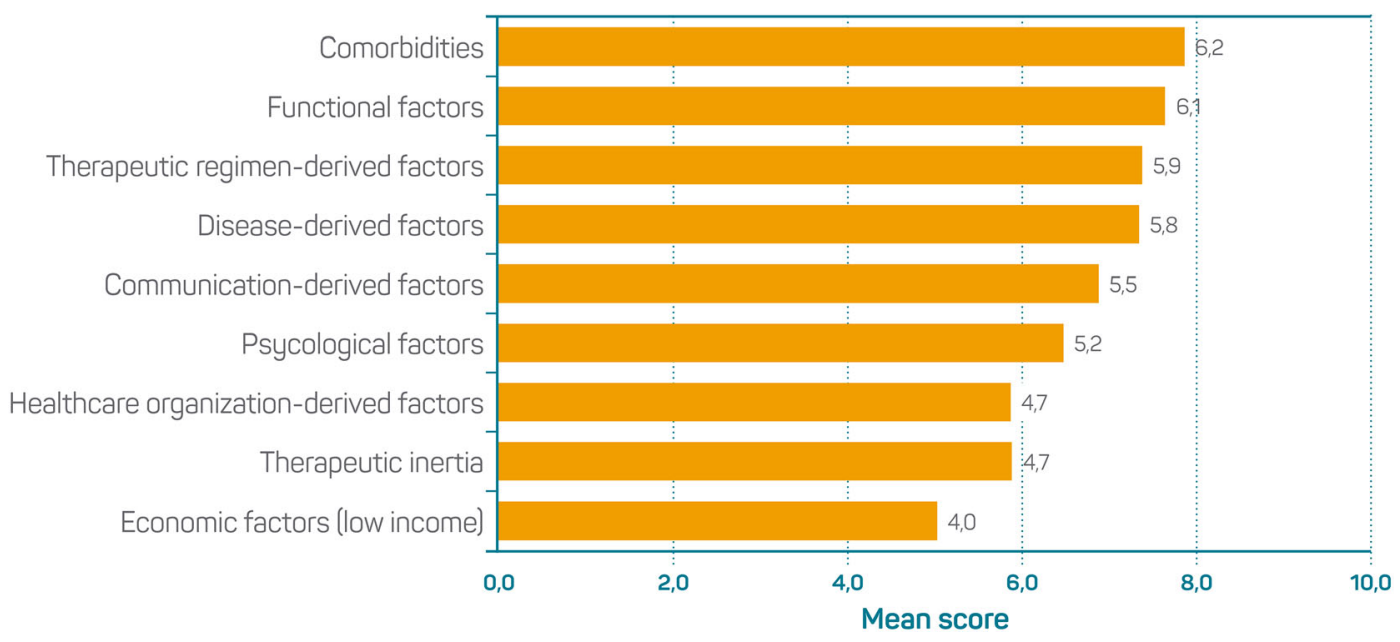

Fig. 2 Questions on opinion, attitude and behaviour (OAB) regarding strict glycaemic control $(N=296)$

rather than a stepwise approach in order to extend the time to treatment failure [31, 32]. The VERIFY study was the first study to show that early intervention with a combination of hypoglycaemic agents as the first therapeutic step provides greater and longer-lasting benefits than a sequential approach: both the incidence of initial treatment failure and the time to treatment failure were significantly lower with the early combination of metformin and vildagliptin than with the sequential treatment regimen, whilst both arms were equally safe and well tolerated [10]. However, similar studies with other antidiabetic molecules, such as dipeptidyl peptidase-4 (DPP-4) inhibitors or those from different drug classes, are needed 


\section{In what situation would you start early treatment intensification? (Multiple choice)}

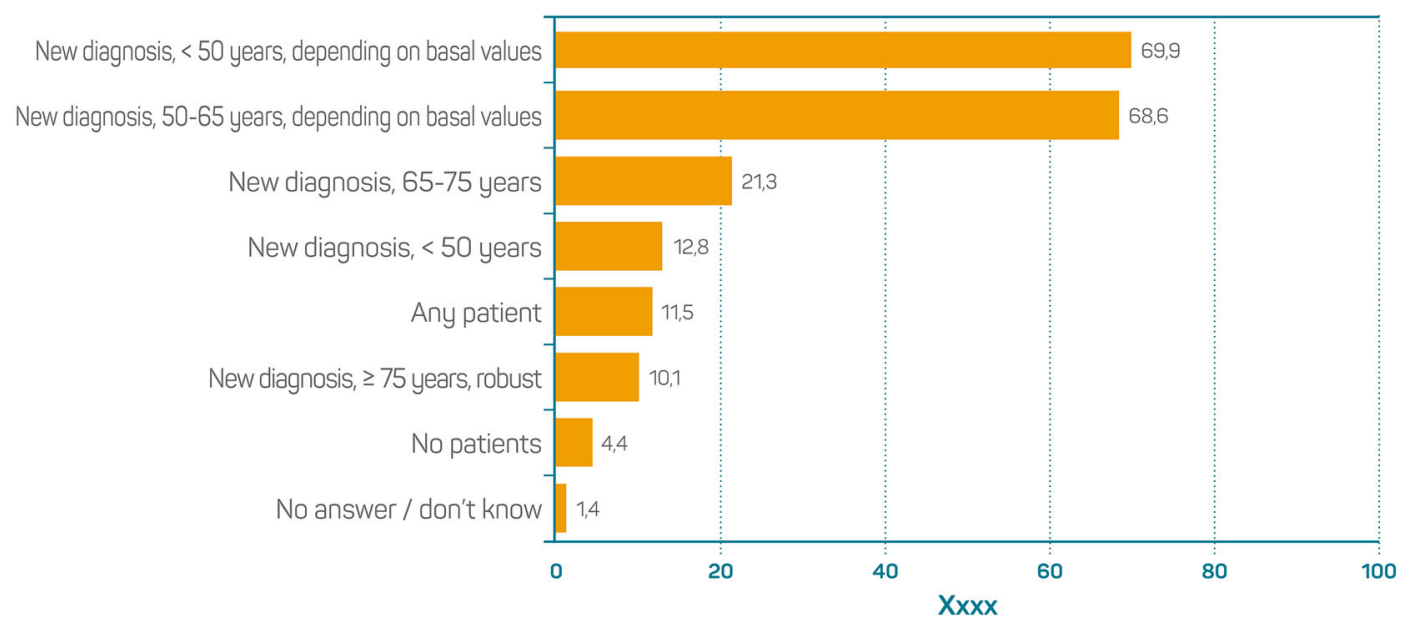

Fig. $3 \mathrm{OAB}$ questions on early treatment intensification $(N=296)$

and would likely result in stronger recommendations and a higher level of (or even universal) consensus on this issue. In this study, agreement the participants showed a high level of agreement when considering the statement on early treatment intensification following diagnosis as a strategy for providing greater and longer-lasting benefits for patients, and the level of agreement was similar across different specialties.

Despite the VERIFY study being performed in patients with a long life expectancy, good functional and cognitive status and recent T2DM diagnosis, $21.3,25.7$ and $27.7 \%$ of participants, respectively, did not believe that these three factors had an influence on individualization towards stricter goals for treatment. Similarly, although the patients included in the VERIFY study benefited from early combined treatment independent of their HbA1c levels, and current international guidelines recommend some antidiabetic classes independently of HbA1c levels in patients with CVD or renal disease [31], almost $70.0 \%$ of the panel considered basal values as the determinant for initiating a combined therapy after the diagnosis, probably prioritizing those patients with higher HbA1c values.

\section{Dysglycaemia}

Continuous glucose monitoring may help to measure and to avoid glycaemic variability and hypoglycaemia [33]. In this study, a large number of participants considered glycaemic variability to be a direct risk factor for the development of chronic complications in patients with T2DM. However, a good level of knowledge of glycaemic variability and of glycaemic monitoring techniques are not widely available, particularly in the PC setting, which could explain the significantly higher agreement amongst HC specialists than PC physicians. As such, the detection and avoidance of glycaemic variability could be an area for improvement.

In line with a number of epidemiological studies [34], postprandial hyperglycaemia was considered to be a CV risk factor by consensus in this study and, consequently, its monitoring and an individualized treatment strategy would be advisable: DPP-4 inhibitors, glucagon-like peptide-1 (GLP-1) receptor agonists and sodium-glucose cotransporter-2 (SGLT-2) inhibitors have been shown to be effective in the reduction of postprandial hyperglycaemia [34-36]. 


\section{a. How do you detect, assess and monitor the risk of hypoglycaemia? (Multiple choice)}

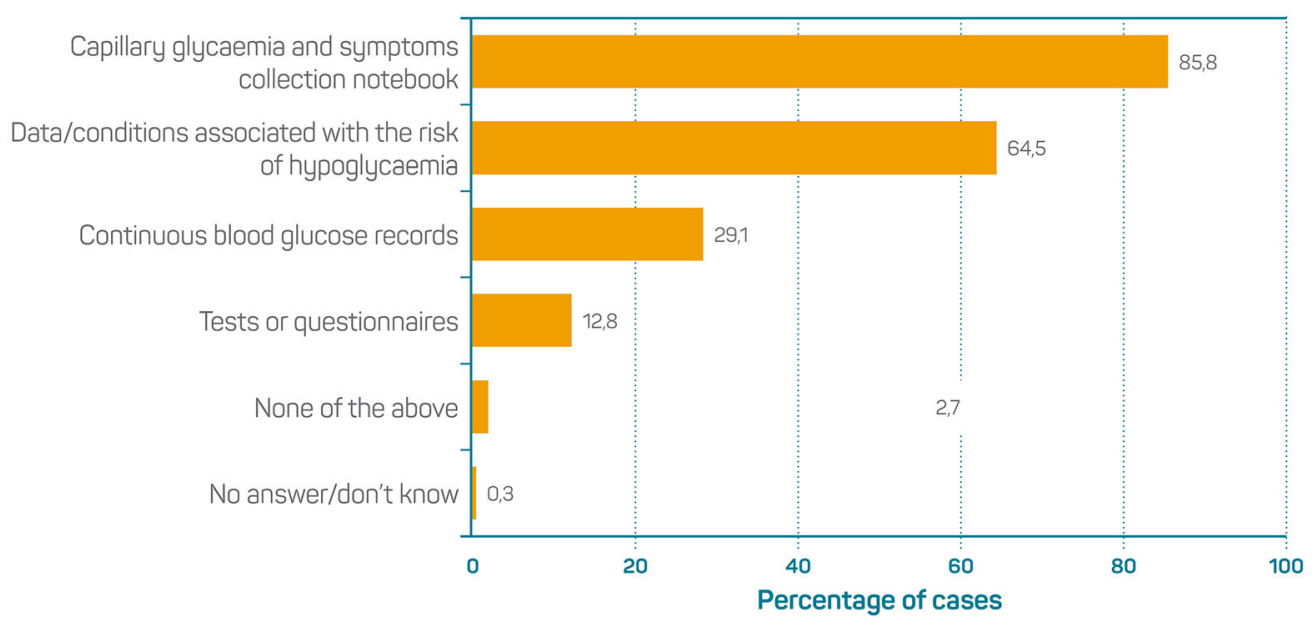

b. In your clinical practice, which situations have you found as a possible cause of hypoglycaemia? (rating scale)

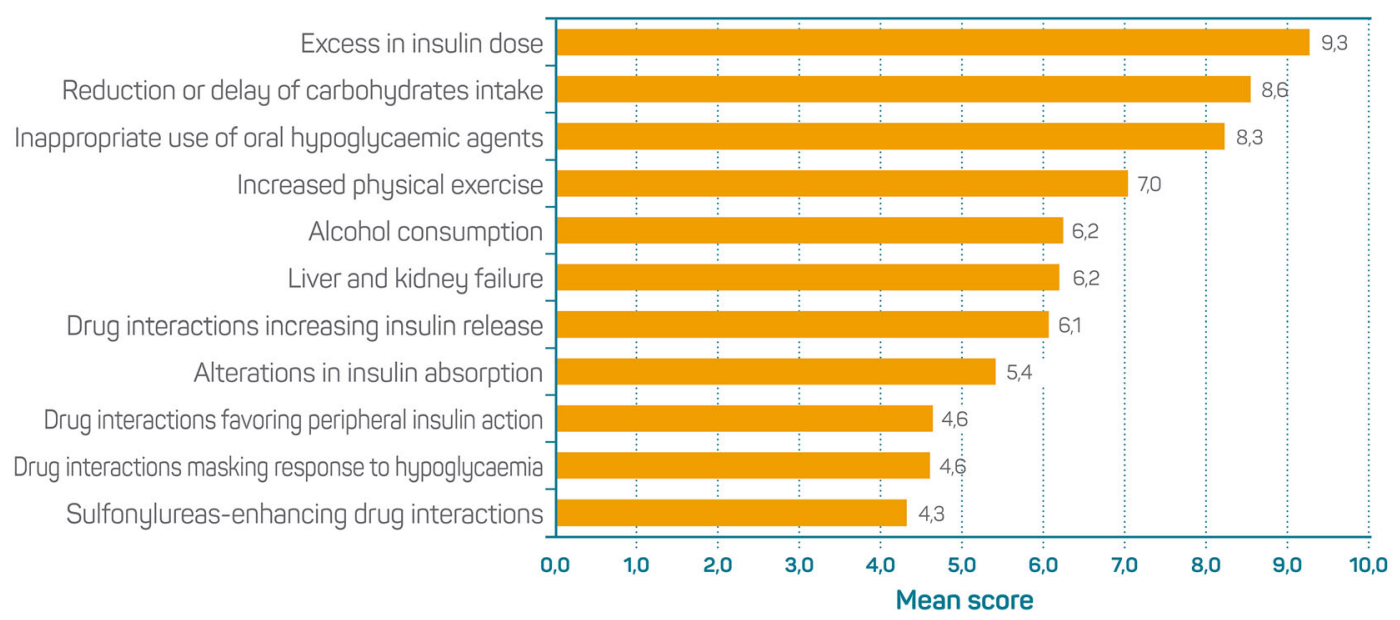

Fig. $4 \mathrm{OAB}$ questions on the risk of hypoglycaemia $(N=296)$

Severe hypoglycaemia is associated with adverse CV outcomes and all-cause mortality, and it is the most important safety concern in patients with diabetes [37]. More than $85.0 \%$ of participants declared assessing and monitoring the risk of hypoglycaemia by measuring capillary glycaemia and recording symptoms, and nearly $65.0 \%$ declared doing so by collecting data and recording associated conditions. In contrast, only a minority seemed to use continuous blood glucose monitoring, tests or questionnaires. The potential risk of severe hypoglycaemia was considered to be a key parameter for defining individualized objectives and choosing a pharmacological treatment for T2DM, with a high degree of agreement. The risk of hypoglycaemia is threefold higher in patients on insulin secretagogues and fivefold higher for those on insulin [38]. In fact, an excessive insulin dose and inappropriate use of 
hypoglycaemic drugs were two of the situations most rated by participants as a cause of hypoglycaemia. The use of newer glucose-lowering agents with minimal risk of hypoglycaemia, such as DPP-4 inhibitors and GLP-1 receptor agonists, can be a useful strategy for avoiding hypoglycaemia [39].

\section{Chronic Complications and Comorbidities}

Almost $80 \%$ of patients with T2DM have comorbidities other than CVD [40]. In light of the findings of the trials completed over the past few years, the independent recommendations from the U.S. Food and Drugs Administration (FDA) and the European Medicines Agency (EMA) for the approval of glucose-lowering molecules for the management of T2DM $[41,42]$ have been recently reviewed, and the inclusion criteria have been broadened to capture a wider range of comorbidities other than CVD amongst the selected patient populations [43]. The presence of chronic complications and comorbidities was considered to interfere with the assessment of the risk-benefit ratio for the available therapeutic options in a higher percentage of HC participants than PC participants. A plausible explanation could be the management of more complex patients in the $\mathrm{HC}$ setting. A better knowledge of the relevance of comorbidities by PC physicians and the selection of options with fewer safety concerns for patients with complications or comorbidities could be two advisable approaches for eliminating these differences.

Psychiatric symptoms are common in patients with T2DM [44, 45], and they worsen disease prognosis, treatment compliance, quality of life and clinical outcomes [46-49]. This also applies to cognitive dysfunction [50, 51]. However, nearly $13.0 \%$ of participants in this study did not express specific agreement when considering psychiatric disorders as comorbidities that affect antidiabetic treatment, with no significant differences between PC physicians and HC specialists.

\section{Limitations of the Study}

This study has a certain number of limitations. First, the sample of participants was selected, although this is a characteristic linked to the Delphi studies in general since they intend to include experts in the field. Second, Delphi questions are an excellent method by which to gain an understanding of opinions on complex situations in clinical practice, but they do not help to explain the reason for the answer given. To counteract this limitation, we included $\mathrm{OAB}$ questions derived from the Delphi statements in the survey. In addition to adding qualitative information, these $\mathrm{OAB}$ questions helped to check coherence and provided robustness to the methodology. The use of closed answers in $\mathrm{OAB}$ questions can lead to a certain bias due to having ignored other answer options. However, those options considered to be more relevant or frequent at the discretion of the scientific committee were included, and the option of including more answers or using an open field would have meant a questionnaire that was too long and the risks of excessive dispersion of the answers. There is the possibility of variable interpretation of the statements or response options according to each participant but, in any case, the questionnaire was reviewed by experts belonging to both the $\mathrm{PC}$ and the $\mathrm{HC}$ fields.

Last but not least, the study has detected a high degree of agreement amongst PC physicians and HC specialists in relation to knowledge of T2DM management, while differences in behaviour could be present due to the different clinical outcomes of patients addressed by each specialty. Therefore, existing mismatches between knowledge and clinical behaviour could have not been detected. In this regard, we suggest that further studies be conducted that highlight the potential behavioural differences between PC physicians and HC specialists in clinical practice.

\section{CONCLUSIONS}

Although high levels of agreement were obtained on all the issues assessed, this study 
shows that there is still room for improvement in terms of implementing strict glycaemic control, individualizing glycaemic control goals and opting for early treatment intensification, with most improvement to be gained amongst PC physicians. Further studies to evaluate the consequences of the lack of glycaemia control and the benefits of early treatment intensification with combinations of glucose-lowering molecules are necessary, and the findings may possibly contribute to achieving higher levels of agreement among physicians and specialists and a transformation of clinical practice.

\section{ACKNOWLEDGEMENTS}

The authors would like to extend special thanks to the panel of primary and hospital care physicians for their participation; to Esteve Pharmaceuticals, S.A for the support provided to carry out this study; and to IDEMM-FARMA S.L for the technical and methodological support.

Funding. Financial and logistic support was provided by ESTEVE Pharmaceuticals S.A Barcelona, Spain, including the journal's Rapid Service Fee. ESTEVE Pharmaceuticals S.A did not influence and was not involved in data interpretation and analysis.

Medical Writing and Other Assistance. The authors would like to thank Montse Fontboté and Jemina Moretó (freelance) for providing medical writing support; and to Francisco López (Acoiba S.L) for statistical assistance. The medical writing and statistical assistance fees were provided by ESTEVE Pharmaceuticals S.A Barcelona, Spain.

Authorship. All named authors meet the International Committee of Medical Journal Editors (ICMJE) criteria for authorship for this article. All named authors take responsibility for the integrity of the work as a whole and have given their approval for this version to be published.
Author Contributions. All authors have equally collaborated on the design of the study, interpretation of data and drafting and review of the manuscript.

Disclosures. Francesc Xavier Cos has taken part in advisory panels; has acted as a speaker for Astra Zeneca, Boehringer Ingelheim, Eli Lilly, Novartis, Novo Nordisk and Sanofi; and has been an investigator in clinical trials for Astra Zeneca, Novartis, Sanofi and Boehringer Ingelheim. Ricardo Gómez-Huelgas has taken part in advisory panels, lectures and studies for Boehringer Ingelheim, Eli Lilly, Novo Nordisk, Sanofi, Astra Zeneca, MSD, Janssen and Esteve. Fernando Gomez-Peralta has taken part in advisory panels for Abbott, Astra Zeneca, Esteve, Novartis, Novo Nordisk and Sanofi; has also been an investigator in clinical trials for Boehringer Ingelheim, Eli Lilly, Novo Nordisk and Sanofi; and has acted as a speaker for Abbott, Astra Zeneca, Boehringer Ingelheim, Bristol Myers Squibb, Eli Lilly, Esteve, Novartis, Novo Nordisk and Sanofi.

Compliance with Ethics Guidelines. The study was based on an on-line survey that did not require data on individual patients to be recorded nor involve the participation of patients. There was no evaluation of any specific medication as the main factor. Therefore, this study did not require ethical approval as none of the criteria of post-authorization studies (PASS, non-interventional PASS) covered by the Spanish Agency for Medicines and Health Products (AEMPS) were met. Data were collected by means of anonymous questionnaires in online format, completed by physicians in accordance with their usual practice. Participation was voluntary. The respondents expressed their consent to participate in the survey through logging into the secure online survey platform and actively clicking a consent box.

Data Availability. The datasets generated during and/or analysed during the current study are available from the corresponding author on reasonable request. 
Open Access. This article is licensed under a Creative Commons Attribution-NonCommercial 4.0 International License, which permits any non-commercial use, sharing, adaptation, distribution and reproduction in any medium or format, as long as you give appropriate credit to the original author(s) and the source, provide a link to the Creative Commons licence, and indicate if changes were made. The images or other third party material in this article are included in the article's Creative Commons licence, unless indicated otherwise in a credit line to the material. If material is not included in the article's Creative Commons licence and your intended use is not permitted by statutory regulation or exceeds the permitted use, you will need to obtain permission directly from the copyright holder. To view a copy of this licence, visit http://creativecommons.org/licenses/by$\mathrm{nc} / 4.0 /$.

\section{REFERENCES}

1. Aschner P, Beck-Nielsen $H$, Bennett $P$, et al. Global guideline for type 2 diabetes. Diabetes Res Clin Pract. 2014;104:1-52.

2. Gómez-Peralta F, Abreu C, Cos X, Gómez-Huelgas R. When does diabetes start? Early detection and intervention in type 2 diabetes mellitus. Rev Clin Esp. 2020;220:305-14.

3. Ceriello A, Gavin JR, Boulton AJM, et al. The Berlin Declaration: a call to action to improve early actions related to type 2 diabetes. How can specialist care help? Diabetes Res Clin Pract. 2018;139: 392-9.

4. Einarson TR, Acs A, Ludwig C, Panton UH. Prevalence of cardiovascular disease in type 2 diabetes: a systematic literature review of scientific evidence from across the world in 2007-2017. Cardiovasc Diabetol. 2018;2018:17.

5. Schnell O, Standl E, Cos X, et al. Report from the 5 th cardiovascular outcome trial (CVOT) summit. Cardiovasc Diabetol. 2020;19:1-10.

6. Phung OJ, Sobieraj DM, Engel SS, Rajpathak SN. Early combination therapy for the treatment of type 2 diabetes mellitus: systematic review and meta-analysis. Diabetes, Obes Metab. 2014;16: 410-7.
7. Alberti KGMM, Zimmet P, Shaw J. International diabetes federation: a consensus on Type 2 diabetes prevention. Diabet Med. 2007;24(5):451-63. https://doi.org/10.1111/j.1464-5491.2007.02157.x.

8. Saran R, Li Y, Robinson B, Ayanian J, Balkrishnan R, Bragg-Gresham J, Chen JTL, Cope E, Gipson D, He K, Herman W, Heung M, Hirth RA, Jacobsen SS, Kalantar-Zadeh K, Kovesdy CP, Leichtman AB, Lu Y, Molnar MZ, Morgenstern H, Nallamothu B, O'Hare AM, Pisoni R, Plattner B, Port FK, Rao P, Rhee CM, Schaubel DE, Selewski DT, Shahinian V, Sim JJ, Song P, Streja E, Kurella Tamura M, Tentori F, Eggers PW, Agodoa LYC, Abbott KC. US Renal Data System 2014 Annual Data Report: Epidemiology of Kidney Disease in the United States. Am J Kidney Dis. 2015;66(1):A7. https://doi.org/10.1053/j.ajkd. 2015.05.001.

9. Bourne RRA, Stevens GA, White RA, Smith JL, Flaxman SR, Price H, Jonas JB, Keeffe J, Leasher J, Naidoo K, Pesudovs K, Resnikoff S, Taylor HR. Causes of vision loss worldwide 1990-2010: a systematic analysis. Lancet Glob Health. 2013;1(6): e339-49. https://doi.org/10.1016/S2214-109X(13) 70113-X.

10. Matthews DR, Paldánius PM, Proot P, Chiang YT, Stumvoll M, Del Prato S. Glycaemic durability of an early combination therapy with vildagliptin and metformin versus sequential metformin monotherapy in newly diagnosed type 2 diabetes (VERIFY): a 5-year, multicentre, randomised, double-blind trial. Lancet. 2019;394:1519-29.

11. Cos X, Seidu S, Brunton S, et al. Impact on guidelines: the general practitioner point of view. Diabetes Res Clin Pract. 2020;166:108091.

12. Buse JB, Wexler DJ, Tsapas A, et al. Update to: Management of hyperglycemia in type 2 diabetes, 2018. A consensus report by the American Diabetes Association (ADA) and the European Association for the Study of Diabetes (EASD). Diabetes Care. 2018;2020(43):487-93.

13. Davies MJ, D'Alessio DA, Fradkin J, et al. Management of hyperglycemia in type 2 diabetes, 2018. A consensus report by the American Diabetes Association (ADA) and the European Association for the Study of Diabetes (EASD). Diabetes Care. 2018;2018(41):2669-701.

14. Mata-Cases M, Franch-Nadal J, Real J, et al. Therapeutic inertia in patients treated with two or more antidiabetics in primary care: factors predicting intensification of treatment. Diabetes Obes Metab. 2018;20:103-12.

15. Vernet Vernet M, Sender Palacios MJ, Bautista Galí L, Larrosa Sàez P, Vargas SJ. Therapeutic inertia in 
the management of type 2 diabetic patients in primary health care. SEMERGEN. 2016;42:152-7.

16. Khunti K, Gomes MB, Pocock S, et al. Therapeutic inertia in the treatment of hyperglycaemia in patients with type 2 diabetes: a systematic review. Diabetes Obes Metab. 2018;20:427-37.

17. Khunti K, Wolden ML, Thorsted BL, Andersen M, Davies MJ. Clinical inertia in people with type 2 diabetes: a retrospective cohort study of more than 80,000 people. Diabetes Care. 2013;36:3411-7.

18. Mehta RR, Edwards AM, Rajpathak S, Sharma A, Snow KJ, Iglay K. Effects of conformance to type 2 diabetes guidelines on health care resource utilization, clinical outcomes, and cost: a retrospective claims analysis. J Clin Transl Endocrinol. 2020;19: 100215.

19. Alkhiari R, Alzayer H, Aljazeeri J, Vanniyasingam T, Punthakee Z. Adherence to guidelines for inpatient pharmacologic management of type 2 diabetes in adults and glycemic outcomes. Can J Diabetes. 2018;42:158-62.

20. Chen Y, Sloan FA, Yashkin AP. Adherence to diabetes guidelines for screening, physical activity and medication and onset of complications and death. J Diabetes Compl. 2015;29:1228-33.

21. Álvarez-Guisasola F. Glycaemic control and implementation of the ADA/EASD-2006 consensus algorithm in type 2 diabetes mellitus patients in primary care in Spain. Int J Clin Pract. 2014;68: 28-39.

22. Tastle JM, Tastle WJ. Extending the consensus measure: Analyzing ordinal data with respect to extrema. Inf Syst Educ J. 2006;2006:4.

23. Serrano R, Javier García-Soidán F, Díaz-Redondo A, et al. Cohort study in primary health care on the evolution of patients with prediabetes (PREDAPS): basis and methodology. Rev Esp Salud Publ. 2013;87:121-35.

24. World Health Organization (WHO). Screening for type 2 diabetes. Report of a World Health Organization and International Diabetes Federation meeting. 2003. https://apps.who.int/iris/bitstream/ handle/10665/68614/WHO_NMH_MNC_03.1.pdf? sequence=1\&isAllowed=y. Accessed 25 May 2021.

25. World Health Organization (WHO). Use of glycated haemoglobin (HbA1c) in the diagnosis of diabetes mellitus. Abbreviated report of a WHO consultation. 2011. https://www.who.int/diabetes/ publications/report-hba1c_2011.pdf. Accessed 25 May 2021.
26. Selph S, Dana T, Blazina I, Bougatsos C, Patel H, Chou R. Screening for type 2 diabetes mellitus: a systematic review for the US preventive services task force. Ann Intern Med. 2015;162:765-76.

27. Turner R. Effect of intensive blood-glucose control with metformin on complications in overweight patients with type 2 diabetes (UKPDS 34). Lancet. 1998;352:854-65.

28. Paul SK, Klein K, Thorsted BL, Wolden ML, Khunti K. Delay in treatment intensification increases the risks of cardiovascular events in patients with type 2 diabetes. Cardiovasc Diabetol. 2015;14:1-10.

29. Laiteerapong N, Ham SA, Gao Y, et al. The legacy effect in type 2 diabetes:impactofearlyglycemic control on future complications (the Diabetes \& Aging Study). Diabetes Care. 2019;42:416-26.

30. Mata-Cases M, Franch-Nadal J, Real J, Mauricio D. Glycaemic control and antidiabetic treatment trends in primary care centres in patients with type 2 diabetes mellitus during 2007-2013 in Catalonia: a population-based study. BMJ Open. 2016;2016:6.

31. American Diabetes Association. Pharmacologic approaches to glycemic treatment: standards of medical care in diabetes-2021. Diabetes Care. 2021;44:S111-24.

32. Seidu S, Cos X, Brunton S, et al. A disease state approach to the pharmacological management of Type 2 diabetes in primary care: a position statement by Primary Care Diabetes Europe. Prim Care Diabetes. 2021;2021:31-51.

33. Gomez-Peralta F, Dunn T, Landuyt K, Xu Y, MerinoTorres JF. Flash glucose monitoring reduces glycemic variability and hypoglycemia: real-world data from Spain. BMJ Open Diabetes Res Care. 2020;8: e001052.

34. Pinés Corrales PJ, Bellido-Castañeda V, AmpudiaBlasco FJ. Update on postprandial hyperglycaemia: the pathophysiology, prevalence, consequences and implications of treating diabetes. Rev Clin Esp. 2020;2020:57-68.

35. Monnier L, Colette C, Owens D. Glucose variability: do we have to revisit the profusion of definitions to avoid confusion? Diabetes Metab. 2018;2018: 97-100.

36. Guerci B, Monnier L, Serusclat P, et al. Continuous glucose profiles with vildagliptin versus sitagliptin in add-on to metformin: results from the randomized Optima study. Diabetes Metab. 2012;38: 359-66.

37. Amiel SA, Aschner P, Childs B, et al. Hypoglycaemia, cardiovascular disease, and mortality in 
diabetes: epidemiology, pathogenesis, and management. Lancet Diabetes Endocrinol. 2019;2019: 385-96.

38. Dunkley AJ, Fitzpatrick C, Gray LJ, et al. Incidence and severity of hypoglycaemia in type 2 diabetes by treatment regimen: a UK multisite 12-month prospective observational study. Diabetes Obes Metab. 2019;21:1585-95.

39. Farngren J, Ahrén B. Incretin-based medications (GLP-1 receptor agonists, DPP-4 inhibitors) as a means to avoid hypoglycaemic episodes. Metabolism. 2019;99:25-31.

40. Mata-Cases M, Franch-Nadal J, Real J, Cedenilla M, Mauricio D. Prevalence and coprevalence of chronic comorbid conditions in patients with type 2 diabetes in Catalonia: a population-based crosssectional study. BMJ Open. 2019;9:e031281.

41. European Medicines Agency. Guideline on clinical investigation of medicinal products in the treatment or prevention of diabetes mellitus. 2012. https:// www.ema.europa.eu/en/documents/scientific-guideline/guideline-clinical-investigation-medicinal-products-treatment-prevention-diabetes-mellitus-revision_en.pdf. Accessed 13 Oct 2020.

42. US Food and Drug Administration. Guidance for industry on diabetes mellitus-evaluating cardiovascular risk in new antidiabetic therapies to Treat type 2 diabetes. 2008. https://www.federalregister. gov/documents/2008/12/19/E8-30086/guidance-forindustry-on-diabetes-mellitus-evaluating-cardiovascular-risk-in-new-antidiabetic. Accessed 13 Oct 2020.

43. Sharma A, Pagidipati NJ, Califf RM, et al. Impact of regulatory guidance on evaluating cardiovascular risk of new glucose-lowering therapies to treat type 2 diabetes mellitus: lessons learned and future directions. Circulation. 2020;2020:843-62.
44. Dogan B, Oner C, Akalin AA, Ilhan B, Caklili OT, Oguz A. Psychiatric symptom rate of patients with diabetes mellitus: a case control study. Diabetes Metab Syndr Clin Res Rev. 2019;13:1059-63.

45. Thomas J, Jones G, Scarinci I, Brantley P. A descriptive and comparative study of the prevalence of depressive and anxiety disorders in lowincome adults with type 2 diabetes and other chronic illnesses. Diabetes Care Diabetes Care. 2003;26:2311-7.

46. Gonzalez JS, Peyrot M, McCarl LA, et al. Depression and diabetes treatment nonadherence: a metaanalysis. Diabetes Care. 2008;31:2398-403.

47. Baumeister H, Hutter N, Bengel J, Härter M. Quality of life in medically Ill persons with comorbid mental disorders: a systematic review and metaanalysis. Psychother Psychosom. 2011;80:275-86.

48. Egede LE, Nietert PJ, Zheng D. Depression and allcause and coronary heart disease mortality among adults with and without diabetes. Diabetes Care. 2005;28:1339-45.

49. Rohde C, Knudsen JS, Schmitz N, Østergaard SD, Thomsen RW. The impact of hospital-diagnosed depression or use of antidepressants on treatment initiation, adherence and $\mathrm{HbA1c} / \mathrm{LDL}$ target achievement in newly diagnosed type 2 diabetes. Diabetologia. 2021;64:361-74.

50. Biessels GJ, Despa F. Cognitive decline and dementia in diabetes mellitus: mechanisms and clinical implications. Nat Rev Endocrinol. 2018;14: 591-604.

51. Gómez-Huelgas R, Gómez Peralta F, Rodríguez Mañas L, et al. Treatment of type 2 diabetes mellitus in elderly patients. Rev Esp Geriatr Gerontol. 2018;53:89-99. 